\title{
Integer-valued Time Series Model via Generalized Linear Models Technique of Estimation
}

\author{
Rasaki Olawale Olanrewaju* \\ Department of Statistics, Distance Learning Center (DLC), University of Ibadan, Nigeria \\ * Corresponding author email: rasakiolawale@gmail.com
}

Received: 27 February 2018 / Revised : 12 April 2018 / Accepted: 15 April 2018 / Published: 29 April 2018

\begin{abstract}
The paper authenticated the need for separate positive integer time series model(s). This was done from the standpoint of a proposal for both mixtures of continuous and discrete time series models. Positive integer time series data are time series data subjected to a number of events per constant interval of time that relatedly fits into the analogy of conditional mean and variance which depends on immediate past observations. This includes dependency among observations that can be best described by Generalized Autoregressive Conditional Heteroscedasticity (GARCH) model with Poisson distributed error term due to its positive integer defined range of values. As a result, an integer GARCH model with Poisson distributed error term was formed in this paper and called Integer Generalized Autoregressive Conditional Heteroscedasticity (INGARCH). Iterative Reweighted Least Square (IRLS) parameter estimation technique type of the Generalized Linear Models (GLM) was adopted to estimate parameters of the two spilt models; Linear and Log-linear INGARCH models deduced from the identity link function and logarithmic link function, respectively. This resulted from the log-likelihood function generated from the GLM via the random component that follows a Poisson distribution. A study of monthly successful bids of auction from 2003 to 2015 was carried out. The Probabilistic Integral Transformation (PIT) and scoring rule pinpointed the uniformity of the linear INGARCH than that of the log-linear INGARCH in describing first order autocorrelation, serial dependence and positive conditional effects among covariates based on the immediate past. The linear INGARCH model outperformed the log-linear INGARCH model with $(\mathrm{AIC}=10514.47, \mathrm{BIC}=10545.01$, QIC $=$ 34128.56) and $(\mathrm{AIC}=37588.83, \mathrm{BIC}=37614.28, \mathrm{QIC}=37587.3)$, respectively.
\end{abstract}

Keywords: Count; Generalized Linear Model (GLM); Integer Generalized Autoregressive Conditional Heteroscedasticity (INGARCH); Iterative Reweighted Least Square (IRLS); Poisson.

\section{Introduction}

Regression time series models for linear models such as Generalized Autoregressive Conditional Heteroscedasticity (GARCH), Autoregressive Moving Average (ARMA) etc. and non-linear models such as Self-Exciting Threshold Autoregressive (SETAR); Mixture Autoregressive (MAR) etc. has been dealt with in many studies. Typically, this is either by way of applying these models to timely related data or by compounding with additional parameter(s). These models were proposed or propounded to accommodate, solve and treat mixed time series observations (both continuous and count observations over a constant interval of time) that might arise from all areas of statistics, econometrics, epidemiology, insurance, etc., as stipulated by [1] and [2].

In as much as only positive integer or count time series observations and sometimes due to encounter in our constant interval readings, Exploratory Data Analysis (EDA) and analyses, there is need for time series models to be strictly separated for both continuous linear and nonlinear models; and count (positive integer) linear and non-linear models without subjecting integer or positive integer-valued related observations to 
a strictly continuous model or vice-versa [3]. Few research and brainstorming have been channeled into count time series analyzes. Tobias $e t$ al. [1] explained that count time series are series of observations that appear naturally in various areas such that a number of events per constant time period is observed over a timeframe e.g. number of cars that entered a particular filling station hourly, number of stock market transactions daily, number of defective items in a production daily, weekly number of admissions in a hospital etc.

It is also to be noted that such observations would be considered as natural values, that is, $y_{t} \in \mathbb{Z}^{+}$. In this regard, some authors, [4] - [6], propound models for integer time series. Models for count data are not only proposed because they take into account suitable dependence among observations but also owing to the fact that they are models whose conditional mean depends on previous observations and immediate past values, [5]. According to [7] - [10], count time series models can be otherwise called state space models because of their dependence traits - and because of this dependence traits among observations, GARCH will be a suitable model in describing and incorporating the analogy of conditional mean and variance depending on immediate past readings possessed by count data, [11], [12].

Having said this, a befitting distribution with an inclusion of non-negativity (positive integer) defined range of positive values, timedependence covariates, linear and non-linear model for positive conditional mean and variance process would be ideal to take in place the conventional Gaussian distribution of the white noise. In lieu of this, the Poisson and Negative
Binomial distribution are best to fill this vacuum [13], but the Poisson distribution would be used due to its simplicity, possessed assumptions of count data depending on time and being among the family of an exponential family [14]:

$$
P\left(\lambda_{t}\right)=\frac{\lambda_{t}^{y} e^{\left(-\lambda_{t}\right)}}{y !} \quad y=0,1, \cdots \cdots
$$

Incorporating equation (1) into the known GARCH model as its error term makes it the Integer Generalized Autoregressive Conditional Heteroscedasticity (INGARCH) of the same " $p$ " and " $q$ ", that is INGARCH (p, q); in other words, GARCH for Count (Integer) Data. The ductile approach ideal to be employed for modeling and estimating parameters of the count time series data conditioned on the past immediate information will be by Generalized Linear Model (GLM) methodology, [15].

\section{Generalized Methodology of the INGARCH Model.}

GLM encompasses General Linear Model (meant for only Gaussian distribution and continuous data); all form of data type (be it discrete count, categorical, binary, etc.) and all forms of distributions of [16]. GLM was implemented to work out appropriate linear predictor link function (mean function), and inverse link function. This was with a view to describing the covariate effects attached to the dependence observations. Whilst imitating the structure-like of this GLM and known GARCH, this paper will mimic the Iterative Reweighted Least Square (IRSL) of one of GLM techniques to estimate parameters in the INGARCH model to explain its variability.

Let $Y_{t} \in \mathbb{Z}^{+}$denote a count time series and $X_{t} \in \mathbb{Z}^{+}$denote a time-varying n-dimensional covariate vector, $X_{t}=\left(X_{t, 1}, X_{t, 2}, \cdots, X_{t, n}\right)^{T}$ with conditional mean $E\left(Y_{t} / F_{t-1}\right)=\lambda_{t} \ni \quad t \in \mathbb{Z}^{+}$where $F_{t}$ the history of joint process is $\left\{Y_{t}, \lambda_{t}, X_{t+1}: t \in \mathbb{Z}^{+}\right\}$up to time " $t$ " including the covariate information at time $" t+1$ ". The INGARCH model from the idea of GARCH is:

$$
h\left(\lambda_{t}\right)=\beta_{0}+\sum_{g=1}^{p} \beta_{g} h\left(Y_{t-i g}\right)+\sum_{d=1}^{q} \alpha_{d} h\left(\lambda_{t-j d}\right)+\eta^{T} X_{t}
$$


Rasaki Olawale Olanrewaju., Int. Ann. Sci.; Vol. 4, Issue 1, pp: 35-43, 2018

$\operatorname{INGARCH}(p, q)=g(\mu)=h\left(\lambda_{t}\right) \Rightarrow$ The link function, $h\left(\lambda_{t}\right)=\mu \Rightarrow$ linear $\quad$ Predictor, $h^{-1}\left(\lambda_{t}\right)=\mu \Rightarrow$ Inverse link function with parameter vector $\eta=\left(\eta_{1}, \eta_{2}, \cdots, \eta_{n}\right)^{T}$, with arbitrary past observations of the independents $P=\left\{i_{1}, i_{2}, \ldots, i_{p}\right\}$ regress on $Y_{t-i 1}, Y_{t-i 2}, \ldots, Y_{t-i p}$ defined on $Q=\left\{j_{1}, j_{2}, \ldots, j_{q}\right\}$ for integers $0<i_{1}<i_{2}<\cdots<i_{p}<\infty ; 0<j_{1}<j_{2}<\cdots<j_{p}<\infty \ni p, q \in \mathbb{Z}^{+}$for any choosing " $p$ " and " $q$ " suitable for $P=\{1, \ldots, p\}, Q=\{1, \ldots, q\}$.

Considering a situation or scenario where the link function in model in equation (2) is identity, that is $h(\mu)=h(\mu)=g\left(\mu_{i}\right)=\mu_{i}=\lambda_{t} \approx \operatorname{Poisson}\left(\lambda_{t}\right)$ such that $\frac{\partial h\left(\lambda_{t}\right)}{\partial \lambda_{t}}=1 ; \eta=0$, gives

$$
\lambda_{t}=\beta_{0}+\sum_{g=1}^{p} \beta_{g} Y_{t-g}+\sum_{d=1}^{q} \alpha_{d} \lambda_{t-d}
$$

For $Y_{t}$ of past observation that is Poisson distributed. equation (3) is the INGARCH (p, q) for LINEAR MODEL.

If model in eqn. (1) has a logarithmic link function, that is $h\left(\lambda_{t}\right)=\log \left(\lambda_{t}\right)$, then $h\left(Y_{t}\right)=\log \left(Y_{t}+1\right)$, by setting $v_{t}=\log \left(\lambda_{t}\right), \frac{\partial h\left(v_{t}\right)}{\partial v_{t}} \neq 1 ; \eta \neq 0$ gives

$$
v_{t}=\beta_{0}+\sum_{g=1}^{p} \log \beta_{g}\left(Y_{t-g}+1\right)+\sum_{d=1}^{q} \alpha_{d} \lambda_{t-d}+\eta^{T} X_{t}
$$

INGARCH for LOG-LINEAR MODEL of the order (p, q). Equation (4) is the log-likelihood function of the GLM.

\section{Parameter Estimation}

The Iterative Reweighted Least Square (IRSL) parameter estimation technique will be adopted in this work. Let $\Theta=\left\{\beta_{0}, \beta_{1}, \ldots, \beta_{p}, \alpha_{1}, \ldots, \alpha_{q}, \eta_{1}, \ldots \eta_{n} \geq 0: \ni \Theta \in \mathfrak{R}^{+}\right\}$. For stationarity condition and erogidic condition of INGARCH, condition $\sum_{g=1}^{p} \beta_{k}+\sum_{d=1}^{q} \alpha_{d}<1$ for $\beta_{0}>0$ as related to GARCH for linear model in equation (3).

Also,

$\Theta=\left\{\beta_{0}<0,\left|\beta_{1}\right|, \ldots,\left|\beta_{p}\right|,\left|\alpha_{1}\right|, \ldots,\left|\alpha_{q}\right|<1\right\}$ for stationarity condition and erogidic condition of $\operatorname{INGARCH}(\mathrm{p}, \mathrm{q})$, condition $\left|\sum_{g=1}^{p} \beta_{k}+\sum_{d=1}^{q} \alpha_{d}\right|<1$ for the log-linear model.

\subsection{Parameter Estimation of the Log-linear INGARCH Model}

The partial derivations of $\lambda_{t}(\Theta)$ is then $\frac{\partial \lambda_{t}(\Theta)}{\partial \Theta}=h^{\prime}\left(\lambda_{t}(\Theta)\right) \frac{\partial v_{t}(\Theta)}{\partial \Theta}$

$$
\begin{aligned}
& \frac{\partial v_{t}(\Theta)}{\partial \Theta}=\left(\frac{\partial v_{t}(\Theta)}{\partial \beta_{0}}, \frac{\partial v_{t}(\Theta)}{\partial \beta_{1}}, \ldots, \frac{\partial v_{t}(\Theta)}{\partial \eta_{n}}\right)^{T} \\
& \frac{\partial v_{t}(\Theta)}{\partial \beta_{0}}=1+\sum_{d=1}^{q} \alpha_{d} \frac{\partial v_{t-j d(\Theta)}}{\partial \beta_{0}} ; \quad \frac{\partial^{2} v_{t}(\Theta)}{\partial \beta_{0} \partial \beta_{0}}=\sum_{d=1}^{q} \frac{\partial^{2} v_{t-j d(\Theta)}}{\partial \beta_{0} \partial \beta_{0}}=\beta_{0} \beta_{0}
\end{aligned}
$$




$$
\begin{aligned}
& \frac{\partial v_{t}(\Theta)}{\partial \beta_{t}}=h\left(Y_{t-i t}\right) \sum_{d=1}^{q} \alpha_{d} \frac{\partial v_{t-j d(\Theta)}}{\partial \beta_{t}} ; \quad \frac{\partial^{2} v_{t}(\Theta)}{\partial \beta_{t} \partial \beta_{t}}=\sum_{d=1}^{q} \frac{\partial^{2} v_{t-j d(\Theta)}}{\partial \beta_{t} \partial \beta_{t}}=\beta_{t} \beta_{t} \\
& \frac{\partial v_{t}(\Theta)}{\partial \alpha_{t}}=\sum_{d=1}^{q} \alpha_{d} \frac{\partial v_{t-j d(\Theta)}}{\partial \alpha_{t}}+v_{t-j t(\Theta)} ; \quad \frac{\partial^{2} v_{t}(\Theta)}{\partial \alpha_{t} \partial \alpha_{t}}=\sum_{d=1}^{q} \frac{\partial^{2} v_{t-j d(\Theta)}}{\partial \alpha_{t} \partial \alpha_{t}}+\frac{\partial v_{t-j t(\Theta)}}{\partial \alpha_{t}}=\alpha_{t} \alpha_{t} \\
& \frac{\partial v_{t}(\Theta)}{\partial \eta_{t}}=\sum_{d=1}^{q} \alpha_{d} \frac{\partial v_{t-j t(\Theta)}}{\partial \eta_{t}}+X_{t, t} ; \quad \frac{\partial^{2} v_{t}(\Theta)}{\partial \eta_{t} \partial \eta_{t}}=\sum_{d=1}^{q} \frac{\partial^{2} v_{t-j d(\Theta)}}{\partial \eta_{t} \partial \eta_{t}}=\eta_{t} \eta_{t} \\
& \frac{\partial v_{t}(\Theta)}{\partial \beta_{0} \partial \beta_{t}}=\sum_{d=1}^{q} \sum_{d=1}^{q} \alpha_{d}^{2} \frac{\left(\partial v_{t-j d(\Theta)}\right)^{2}}{\partial \beta_{0} \partial \beta_{t}}+\tilde{h}\left(Y_{t-i t}\right) \sum_{d=1}^{q} \alpha_{d} \frac{\partial v_{t-j d(\Theta)}}{\partial \beta_{0}}+\frac{\partial v_{t-j d(\Theta)}}{\partial \beta_{t}}=\beta_{0} \beta_{1} \\
& \frac{\partial v_{t}(\Theta)}{\partial \beta_{0} \partial \alpha_{t}}=\sum_{d=1}^{q} \sum_{d=1}^{q} \alpha_{d}^{2} \frac{\left(\partial v_{t-j d(\Theta)}\right)^{2}}{\partial \beta_{0} \partial \alpha_{t}}+v_{t-j t(\Theta)}\left[1+\sum_{d=1}^{q} \alpha_{d} \frac{\partial v_{t-j d(\Theta)}}{\partial \beta_{0}}\right]+\sum_{d=1}^{q} \alpha_{d} \frac{\partial v_{t-j d(\Theta)}}{\partial \alpha_{t}}=\beta_{0} \alpha_{t} \\
& \frac{\partial v_{t}(\Theta)}{\partial \beta_{0} \partial \eta_{t}}=\sum_{d=1}^{q} \sum_{d=1}^{q} \alpha_{d}^{2} \frac{\left(\partial v_{t-j d(\Theta)}\right)^{2}}{\partial \beta_{0} \partial \eta_{t}}+X_{t, t}\left[1+\sum_{d=1}^{q} \alpha_{d} \frac{\partial v_{t-j d(\Theta)}}{\partial \beta_{0}}\right]+\sum_{d=1}^{q} \alpha_{d} \frac{\partial v_{t-j d(\Theta)}}{\partial \eta_{t}}=\beta_{0} \eta_{t} \\
& \frac{\partial v_{t}(\Theta)}{\partial \beta_{t} \partial \alpha_{t}}=\sum_{d=1}^{q} \sum_{d=1}^{q} \alpha_{d}^{2} \frac{\left(\partial v_{t-j d(\Theta)}\right)^{2}}{\partial \beta_{t} \partial \alpha_{t}}+\tilde{h}\left(Y_{t-i t}\right)\left[v_{t-j t(\Theta)}+\sum_{d=1}^{q} \alpha_{d} \frac{\partial v_{t-j d(\Theta)}}{\partial \alpha_{t}}\right]+v_{t-j t(\Theta)} \sum_{d=1}^{q} \alpha_{d} \frac{\partial v_{t-j d(\Theta)}}{\partial \beta_{t}}=\beta_{t} \alpha_{t} \\
& \frac{\partial v_{t}(\Theta)}{\partial \beta_{t} \partial \eta_{t}}=\sum_{d=1}^{q} \sum_{d=1}^{q} \alpha_{d}^{2} \frac{\left(\partial v_{t-j d(\Theta)}\right)^{2}}{\partial \beta_{t} \partial \eta_{t}}+X_{t, t}\left[v_{t-j t(\Theta)}+\sum_{d=1}^{q} \alpha_{d} \frac{\partial v_{t-j d(\Theta)}}{\partial \alpha_{t}}\right]+v_{t-j t(\Theta)} \sum_{d=1}^{q} \alpha_{d} \frac{\partial v_{t-j d(\Theta)}}{\partial \eta_{t}}=\beta_{t} \eta_{t} \\
& \frac{\partial v_{t}(\Theta)}{\partial \alpha_{t} \partial \eta_{t}}=\sum_{d=1}^{q} \sum_{d=1}^{q} \alpha_{d}^{2} \frac{\left(\partial v_{t-j d(\Theta)}\right)^{2}}{\partial \alpha_{t} \partial \eta_{t}}+X_{t, t}\left[v_{t-j i t(\Theta)}+\sum_{d=1}^{q} \alpha_{d} \frac{\partial v_{t-j d(\Theta)}}{\partial \alpha_{t}}\right]+v_{t-j t(\Theta)} \sum_{d=1}^{q} \alpha_{d} \frac{\partial v_{t-j d(\Theta)}}{\partial \eta_{t}}=\alpha_{t} \eta_{t} \\
& f o r \beta_{t} ; l=1, \ldots, p \\
& \alpha_{t} ; l=1, \ldots, q \\
& \eta_{t} ; l=1, \ldots, n
\end{aligned}
$$$$
\frac{\partial^{2} v_{t}(\Theta)}{\partial \Theta \partial \Theta^{T}}=\left[\begin{array}{cccc}
\beta_{0} \beta_{0} & \beta_{0} \beta_{t} & \beta_{0} \alpha_{t} & \beta_{0} \eta_{t} \\
\beta_{t} \beta_{0} & \beta_{t} \beta_{t} & \beta_{t} \alpha_{t} & \beta_{t} \eta_{t} \\
\alpha_{t} \beta_{0} & \alpha_{t} \beta_{t} & \alpha_{t} \alpha_{t} & \alpha_{t} \eta_{t} \\
\eta_{t} \beta_{0} & \eta_{t} \beta_{t} & \eta_{t} \alpha_{t} & \eta_{t} \eta_{t}
\end{array}\right] \text { for } p=q=n \text { (squarematrix } \text { nby } n \text { ) }
$$

Such that:

Setting

$$
\gamma^{(m)}=\left(\begin{array}{c}
\beta_{0} \\
\beta_{t} \\
\alpha_{t} \\
\eta_{t}
\end{array}\right), \quad \frac{\partial v_{t}(\Theta)}{\partial \Theta}=\left(\begin{array}{c}
\frac{\partial \beta_{0}}{\partial \Theta} \\
\frac{\partial \beta_{t}}{\partial \Theta} \\
\frac{\partial \alpha_{t}}{\partial \Theta} \\
\frac{\partial \eta_{t}}{\partial \Theta}
\end{array}\right)
$$


Adopting the Newton-Raphson multivariate iterative technique: $\gamma^{(m+1)}=\gamma^{(m)}-\left[\frac{\partial^{2} v_{t}(\Theta)}{\partial \Theta \partial \Theta^{T}}\right]^{-1}\left(\frac{\partial v_{t}(\Theta)}{\partial \Theta}\right)$

So, the Fisher Scoring algorithm, $\gamma^{(m+1)}=\gamma^{(m)}+\left[-\mathrm{E}\left(\frac{\partial^{2} v_{t}(\Theta)}{\partial \Theta \partial \Theta^{T}}\right)\right]^{-1}\left(\frac{\partial v_{t}(\Theta)}{\partial \Theta}\right)$

$$
\gamma^{(m+1)}=\gamma^{(m)}+\left[I_{n}^{(m)}\right]^{-1} S^{(m)}
$$

Where $I_{n}^{(m)}$ and $S^{(m)}$ are the Fisher information and Score matrixes respectively to be evaluated by $\gamma^{(m)}=\gamma^{(m+1)}$ via iterative procedure.

\subsection{Parameter Estimation of the Linear INGARCH Model}

The Identity link of the linear predictor $\mathrm{E}\left(v_{t}\right)=\lambda_{t}(\Theta)=\mu(\Theta), \quad \eta=0$

$$
\begin{aligned}
& \frac{\partial \mu(\Theta)}{\partial \Theta}=\left(\frac{\partial v_{t}(\Theta)}{\partial \beta_{0}}, \frac{\partial v_{t}(\Theta)}{\partial \beta_{1}}, \ldots, \frac{\partial v_{t}(\Theta)}{\partial \eta_{n}}\right)^{T} \\
& \frac{\partial \mu(\Theta)}{\partial \beta_{0}}=\frac{1}{1-\sum_{g=1}^{p} \beta_{g}-\sum_{d=1}^{q} \alpha_{d}} ; \frac{\partial \mu(\Theta)}{\partial \beta_{g}}=\frac{\beta_{0}}{\left(1-\sum_{g=1}^{p} \beta_{g}-\sum_{d=1}^{q} \alpha_{d}\right)^{2}} ; \frac{\partial \mu(\Theta)}{\partial \alpha_{d}}=\frac{\beta_{0}}{\left(1-\sum_{g=1}^{p} \beta_{g}-\sum_{d=1}^{q} \alpha_{d}\right)^{2}} \\
& \frac{\partial \mu(\Theta)}{\partial \eta_{n}}=0 ; \quad \frac{\partial \mu(\Theta)}{\partial \beta_{0} \partial \beta_{g}}=\frac{\beta_{0}}{\left(1-\sum_{g=1}^{p} \beta_{g}-\sum_{d=1}^{q} \alpha_{d}\right)^{3}}=\beta_{0} \beta_{g} ; \quad \frac{\partial \mu(\Theta)}{\partial \beta_{0} \partial \eta_{n}}=0=\beta_{0} \eta_{n} \\
& \frac{\partial \mu(\Theta)}{\partial \beta_{0} \partial \alpha_{d}}=\frac{\beta_{0}}{\left(1-\sum_{g=1}^{p} \beta_{g}-\sum_{d=1}^{q} \alpha_{d}\right)^{3}}=\beta_{0} \alpha_{d} ; \quad \frac{\partial \mu(\Theta)}{\partial \beta_{g} \partial \alpha_{d}}=\frac{\beta_{0}^{2}}{\left(1-\sum_{g=1}^{p} \beta_{g}-\sum_{d=1}^{q} \alpha_{d}\right)^{4}}=\beta_{g} \alpha_{d} ; \\
& \frac{\partial \mu(\Theta)}{\partial \beta_{g} \partial \beta_{g}}=\frac{\partial \mu(\Theta)}{\partial \alpha_{d} \partial \alpha_{d}}=\frac{\beta_{0}^{2}}{\left(1-\sum_{g=1}^{p} \beta_{g}-\sum_{d=1}^{q} \alpha_{d}\right)^{4}}=\beta_{g} \beta_{g}=\alpha_{d} \alpha_{d} ; \frac{\partial \mu(\Theta)}{\partial \beta_{0} \partial \beta_{0}}=\frac{1}{\left(1-\sum_{g=1}^{p} \beta_{g}-\sum_{d=1}^{q} \alpha_{d}\right)^{2}}=\beta_{0} \beta_{0} \\
& \frac{\partial \mu(\Theta)}{\partial \beta_{g} \partial \eta_{n}}=\frac{\partial \mu(\Theta)}{\partial \alpha_{d} \partial \beta_{g}}=\frac{\partial \mu(\Theta)}{\partial \alpha_{d} \partial \eta_{n}}=0=\beta_{g} \eta_{n}=\alpha_{d} \beta_{g}=\alpha_{d} \eta_{n} \text { for } \beta_{g}, g=1, \ldots, p ; \alpha_{d}, d=1, \ldots, q \\
& \frac{\partial^{2} \mu(\Theta)}{\partial \Theta \partial \Theta^{T}}=\left[\begin{array}{cccc}
\beta_{0} \beta_{0} & \beta_{0} \beta_{g} & \beta_{0} \alpha_{d} & 0 \\
\beta_{g} \beta_{0} & \beta_{g} \beta_{g} & \beta_{g} \alpha_{d} & 0 \\
\alpha_{d} \beta_{0} & \alpha_{d} \beta_{g} & \alpha_{d} \alpha_{d} & 0 \\
0 & 0 & 0 & 0
\end{array}\right] \text { for } p=q=n(\text { squarematrix } n b y n) \\
& \text { Such that setting } \quad \gamma^{(k)}=\left(\begin{array}{c}
\beta_{0} \\
\beta_{g} \\
\alpha_{d}
\end{array}\right), \quad \frac{\partial \mu(\Theta)}{\partial \Theta}=\left(\begin{array}{c}
\frac{\partial \beta_{0}}{\partial \Theta} \\
\frac{\partial \beta_{g}}{\partial \Theta} \\
\frac{\partial \alpha_{d}}{\partial \Theta}
\end{array}\right)
\end{aligned}
$$


Adopting the Newton-Raphson multivariate iterative technique: $\gamma^{(k+1)}=\gamma^{(k)}-\left[\frac{\partial^{2} \mu(\Theta)}{\partial \Theta \partial \Theta^{T}}\right]^{-1}\left(\frac{\partial \mu(\Theta)}{\partial \Theta}\right)$

So, the Fisher Scoring algorithm, $\gamma^{(k+1)}=\gamma^{(k)}+\left[-\mathrm{E}\left(\frac{\partial^{2} \mu(\Theta)}{\partial \Theta \partial \Theta^{T}}\right)\right]^{-1}\left(\frac{\partial \mu(\Theta)}{\partial \Theta}\right)$

$$
\gamma^{(k+1)}=\gamma^{(k)}+\left[I_{n}^{(k)}\right]^{-1} S^{(k)}
$$

Where $I_{n}^{(k)}$ and $S^{(k)}$ are the Fisher information and Score matrixes respectively to be evaluated by $\gamma^{(k)}=\gamma^{(k+1)}$ via iterative procedure.

\section{Analytical Results and Discussion}

The number of successful bid of government auctions in Nigeria from 2002 to 2015 was used as illustration. The number of auction bids were recorded in a monthly uniform interval by the Central Bank of Nigeria with 156 counted data points over the thirteen (13) years period.

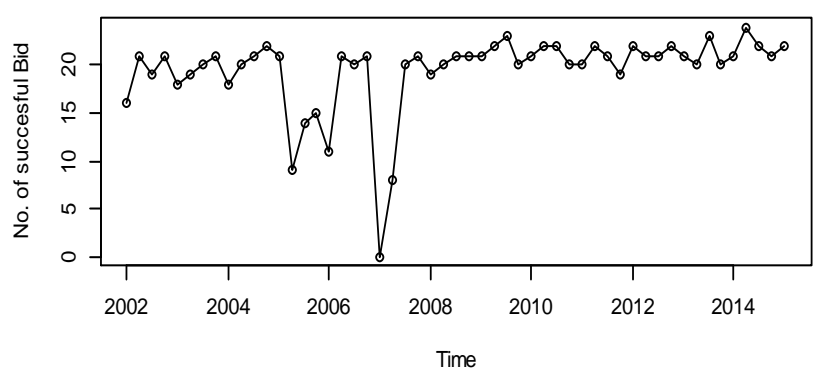

Figure 1: Time plot of the monthly number of successful bids of auctions

From the time plot in Figure 1 above, it was obvious that the number of auction successful bids swing between 10 and 25 monthly expect for a fall in the bid around early 2007. It was unarguably that the number of bids is positive counts monthly.

Apart from the identical autocorrelation function of both the INGARCH log-linear and linear models' residuals, their series were nothing but a realization of discrete white noise as shown in Figure 2. Their autocorrelation residuals indicated a time-dependence effect that do leads to conditional mean and variance process with a short-range serial dependence by a first order autoregressive each. Their squared residuals unveiled the evidence of serial and conditional heteroscedasticity trait present in their timevarying counts.
ACF of the Log-linear

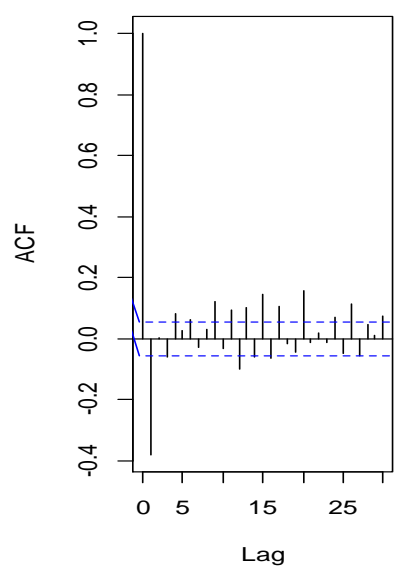

ACF of the linear

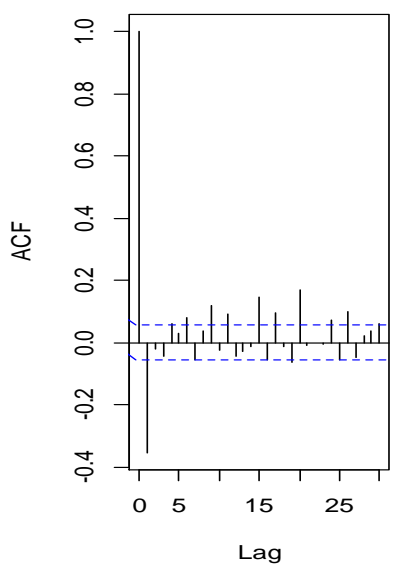

Figure 2: The autocorrelation residuals of the loglinear and linear INGARCH models respectively.

Ascertaining the correctness instrument via the PIT histograms, the histogram bars in the linear INGARCH model appears more clustered as oppose to the disentangled bars (the U-shaped) of the log-linear INGARCH model. The linear INGARCH model seems to approach uniformity better. The probabilistic integral transformation of the linear model seems to be more appropriate.

Table 1: Scoring rules for the linear and loglinearized INGARCH model.

\begin{tabular}{|l|l|l|l|l|l|}
\hline Scores & Logarithmic & Sq. Error & $\begin{array}{l}\text { Norm. Sq. } \\
\text { Error }\end{array}$ & $\begin{array}{l}\text { Ranked } \\
\text { prob. }\end{array}$ & $\begin{array}{l}\text { Dawid- } \\
\text { Sebastiani }\end{array}$ \\
\hline $\begin{array}{l}\text { Log- } \\
\text { linear }\end{array}$ & 15.657845 & 11129.48 & 25.4587788 & 37.42548 & 29.214641 \\
\hline Linear & 4.376028 & 10563.26 & 0.9958283 & 34.65504 & 7.411528 \\
\hline
\end{tabular}

Keys: Sq. error $=$ Squared Error;

Norm. Sq. Error $=$ Normalized Squared Error; prob. $=$ probability

Considering the estimated scoring indexes for the two INGARCH models as shown in Table 1. It can be deduced that the linear INGARCH model 
Rasaki Olawale Olanrewaju., Int. Ann. Sci.; Vol. 4, Issue 1, pp: 35-43, 2018

will capture the time-varying effects in the count observations compare to the log-linear INGARCH model in terms of model performance via logarithmic and ranked probability distribution indexes; via DawidSebastiani index of correcting and penalization of dispersion variation (overestimation and underestimation) through the variance; and via a lower predictive error (integer-valued forecast) of squared error and normalized squared error. The outcome of the scoring rule collaborates with the already established indication by PIT histograms in figure 3. This might be due to non-negative covariates effects and small magnitude of the count series.
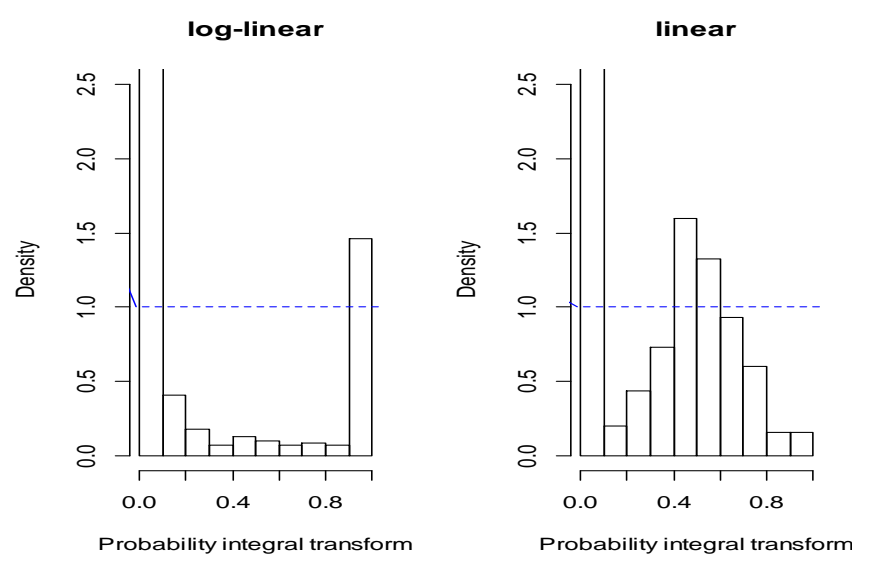

Figure 3: The Probability Integral Transformation (PIT) of the models

Table 2: Coefficients of the linear INGARCH $(1,1)$ model.

\begin{tabular}{|l|l|l|l|l|l|}
\hline Parameters & Estimate & $\begin{array}{l}\text { Std. } \\
\text { Error }\end{array}$ & t-value & $\begin{array}{l}\text { P- } \\
\text { value }\end{array}$ & $\begin{array}{l}\text { 95 \% } \\
\text { C.I }\end{array}$ \\
\hline (Intercept) & 5.4666 & 3.2806 & 16.492 & 0.0000 & $\begin{array}{l}(-0.9631 \\
11.896)\end{array}$ \\
\hline beta_1 & 0.7919 & 0.0931 & 52.201 & 0.0020 & $\begin{array}{l}(0.6094 \\
0.974)\end{array}$ \\
\hline alpha_1 & 0.1711 & 0.0918 & 102.402 & 0.0009 & $\begin{array}{l}(-0.0087 \\
0.351)\end{array}$ \\
\hline interv_1 & 0.0009 & 2.8761 & 15.290 & 0.0000 & $\begin{array}{l}(-5.6361 \\
5.638)\end{array}$ \\
\hline interv_2 & 0.6170 & 6.1979 & 27.390 & 0.0000 & $\begin{array}{l}(- \\
10.5406 \\
13.755)\end{array}$ \\
\hline dispersion & 0.0457 & - & 35.289 & - & - \\
\hline
\end{tabular}

Log-likelihood $=-5251.234 ;$ AIC $=10514.47 ; \mathrm{BIC}=$ 10545.01; QIC = 34128.56

$Y_{t} \sim \operatorname{poisson}\left(\lambda_{t}\right)$

$\lambda_{t}=5.467+0.792 Y_{t-1}+0.171 \lambda_{t-1}+0.000935 X_{t}+0.607 X_{t-12}$ for $t=1, \ldots \ldots, 156$
Table 3: Coefficients of the Log-linear INGARCH $(1,1)$ model

\begin{tabular}{|l|l|l|l|l|l|}
\hline Parameters & Estimate & Std.Error & t-value & P-value & $\begin{array}{c}95 \% \\
\text { CI }\end{array}$ \\
\hline (Intercept) & 9.4230 & 0.4726 & 34.823 & 0.0033 & $\begin{array}{l}(8.5037 \\
10.356)\end{array}$ \\
\hline beta_1 & 0.8453 & 0.0056 & 52.092 & 0.0000 & $\begin{array}{l}(0.8344 \\
0.856)\end{array}$ \\
\hline alpha_1 & 0.1063 & 0.0057 & 64.715 & 0.0000 & $\begin{array}{l}(0.0951 \\
0.118)\end{array}$ \\
\hline interv_1 & 0.0048 & 0.4570 & 5.8912 & 0.0041 & $\begin{array}{l}(-0.8910 \\
0.901)\end{array}$ \\
\hline interv_2 & 0.6003 & 3.3594 & 23.797 & 0.0713 & $\begin{array}{l}(-5.9740 \\
7.195)\end{array}$ \\
\hline
\end{tabular}

Log-likelihood= $-18789.41 ;$ AIC $=37588.83 ;$ BIC = 37614.28; $\mathrm{QIC}=37587.3$

$Y_{t} \sim$ poisson $\left(\log \left(\lambda_{t}\right)\right)$

$\log \left(\lambda_{t}\right)=9.4230+0.845 Y_{t-1}+0.106 \lambda_{t-1}+0.005 X_{t}+0.610 X_{t-12} \quad$ fort $=1, \ldots \ldots, 156$

From table 2 and 3, beta_1 + alpha_1 $=0.963<$ 1 and beta_1 + alpha_1 $=0.9516<1$ for the INGARCH $(1,1)$ linear and $\log$-linear INARCH $(1,1)$ model respectively confirm the presence of positive conditional variability, that is, the stability of the models, with stability realized in the linear INGARCH $(1,1)$ model due to its smaller standard error of the intercept (3.2806) compare to that of in log-linear INGARCH $(1,1)$ model with (0.4726). The alpha_1s of 0.1711 (17.11) and 0.1063 (10.63) in the respectively models explained the level of covariates effects (positive variation) conditioned on the present count based on the immediate past, with a high positive and reliable effect recorded in INGARCH $(1,1)$ linear model. The coefficients of $X_{t} \& X_{t-12}$ explained the effect of the first to last month of every year order autocorrelation in the models. The interv_1 and interv_2 coefficients measure the increment/awareness at the beginning and end of every year, it seems to be more effective in the linear model. Based on the two models fitted, the linear model possessed a robust model performance that best explained covariates effect with smaller (AIC $=10514.47$; $\mathrm{BIC}=10545.01 ; \mathrm{QIC}=34128.56)$ compare to model performance by log-linear model with $(\mathrm{AIC}=37588.83 ; \mathrm{BIC}=37614.28 ; \mathrm{QIC}=$ 37587.3). The Integer-valued and reliable forecasts would be realistic via the linear model compare to the log-linear model. Lastly, an overdispersion of $4.57 \%$ was unfolded, that is the 
variance of the process was 0.0457 greater than its mean.

\section{Conclusions}

In conclusion, count time series model should be associated with discrete distribution(s) such as the Poisson distribution, as the white noise that follows the distributional properties of a timely and positive integer observed series for sufficient and efficient parameters that will truly capture and explain the dependence traits and variability among observations. Having said this, no need of subjecting strictly counts (positive integer) data to the conventional GARCH model with Gaussian independently distributed (distribution for continuous data) error terms when variability and dependence among observations are of interest when considering positive integer observations; instead, INGARCH with Poisson distributed (distribution for positive integer-valued data) error term will be ideal. The study revealed that the INGARCH linear model captured serial and conditional heteroscedasticity in the time varying and time dependent counts (positive integervalued observations) trait present in the of the monthly successful bids of auction than that of the log-linearized INGARCH model. Also unfolded was the first order autocorrelation(lag), that best describe the conspicuous dependence on the number of successful auction bids for in previous month and year with the conditional heteroscedasticity and covariates effects conditioned on the preceding month on the present month.

\section{Declarations}

\subsection{Acknowledgements}

I appreciate the Central bank of Nigeria's research committee for the permission given to use the auction data for research. I thank Mr. Tayo Oladebo and Miss Isamot Omodolapo for their useful and constructive comments; suggestions and insight in the course of the manuscript.

\subsection{Competing Interests}

Author declared, no potential conflict of interest exists.

\section{How to Cite this Article:}

R. Olanrewaju, "Integer-valued Time Series Model via Generalized Linear Models Technique of Estimation", International Annals of Science, vol. 4, no. 1, pp. 35-43, Apr. 2018. doi: 10.21467/ias.4.1.35-43

\section{References}

[1] L. Tobias, K. Fokianos, and R. Fried, "An R Package for Analysis of Count Time Series Following Generalized Linear Models." Journal of Statistical Software. vol.85, no.5, pp. 1-51, Nov. 2017.

[2] M. Salmon, D. Schumacher, and M. Höhle, "Monitoring Count Time Series in R: Aberration Detection in Public Health Surveillance." Journal of Statistical Software, vol. 70, no.10, pp. 1-35, April 2016.

[3] M. S. Christopher, L.B. Robert, L. M. Kerrie, and J. Robert, "PySSM: A Python Module for Bayesian Inference of Linear Gaussian State Space Models", Journal of Statistical Software, vol. 57, no. 6, Mar. 2014.

[4] A. Agosto, G. Cavaliere, D. Kristensen, and A. Rahbek, "Modeling Corporate Defaults: Poisson Autoregressions with Exogenous Covariates (PARX)". CREATES Research Paper School of Economics and Management, University of Aarhus, 2015.

[5] V. Christou, and K. Fokianos, "On Count Time Series Prediction". Journal of Statistical Computation and Simulation, vol. 85, no.2, pp. 357-373, 2015.

[6] H. Elsaied, and R. Fried, "Robust Fitting of INARCH Models". Journal of Time Series Analysis, vol.35, no.6, pp. 517-535, Jun. 2014.

[7] R. Jung, and A. Tremayne, "Useful Models for Time Series of Counts or Simply Wrong Ones?" Advances in Statistical Analysis, vol. 95, no.1, pp. 59-91, 2011.

[8] K. Fokianos, "Count Time Series Models". In T. Subba Rao, S Subba Rao, CR Rao (eds.), Time Series - Methods and Applications, Handbook of Statistics, pp. 315-347, 2012. Elsevier, Amsterdam.

[9] D. Tjøstheim, "Count Time Series with Observation-Driven Autoregressive Parameter Dynamics." In R Davis, S Holan, R Lund, N Ravishanker (eds.), Handbook of Discrete Valued Time Series, Handbooks of Modern Statistical Methods, Chapman \& Hall, London, pp. 77-100, 2015.

[10] M. Yang, J.E. Cavanaugh, and G.K.D. Zamba, "State-Space Models for Count Time Series with Excess Zeros.” Statistical Modeling, vol.15, no.1, pp. 70-90, Nov. 2015. 
Rasaki Olawale Olanrewaju., Int. Ann. Sci.; Vol. 4, Issue 1, pp: 35-43, 2018

[11] P. Brockwell, and R. Davis, "Introduction to Time Series and Forecasting", $2^{\text {nd }}$ edition. SpringerVerlag, New York, 2010.

[12] J. Durbin, and S. Koopman, "Time Series Analysis by State Space Methods." Oxford University Press, Oxford, May 2012.

[13] K. Fokianos, and D. Tjøstheim, "Nonlinear Poisson Auto regression." The Annals of the Institute of Statistical Mathematics, vol. 64, no.6, pp. 1205-1225, Oct. 2012.

[14] K. Fokianos, "Statistical Analysis of Count Time Series Models: A GLM Perspective." In R Davis,
S Holan, R Lund, N Ravishanker (eds.), Handbook of Discrete-Valued Time Series, Handbooks of Modern Statistical Methods, Chapman \& Hall, London, pp. 3-28, May 2015.

[15] J.A. Nelder, and R.W.M. Wedderburn, "Generalized Linear Models". Journal of the Royal Statistical Society, vol.135, no. 3, pp.370384, 1972.

[16] J. Fox, "Maximum-Likelihood Estimation of Generalized Linear Models: Applied Regression Analysis and Generalized Linear Models" (2 ${ }^{\text {nd }}$ Edition). New York: Springer 2008.

\section{Publish your research article in AIJR journals- \\ $\checkmark$ Online Submission and Tracking \\ $\checkmark$ Peer-Reviewed \\ $\checkmark \quad$ Rapid decision \\ $\checkmark \quad$ Immediate Publication after acceptance \\ $\checkmark \quad$ Articles freely available online \\ $\checkmark \quad$ Retain full copyright of your article. \\ Submit your article at journals.aijr.in}

Publish your books with AIJR publisher-

$\checkmark \quad$ Publish with ISBN and DOI.

$\checkmark$ Publish Thesis/Dissertation as Monograph.

$\checkmark \quad$ Publish Book Monograph.

$\checkmark \quad$ Publish Edited Volume/ Book.

$\checkmark \quad$ Publish Conference Proceedings

$\checkmark \quad$ Retain full copyright of your books.

Submit your manuscript at books.aijr.org 